\title{
VARIÁVEIS QUALITATIVAS DA PULVERIZAÇÃo EM FEIJÃO, Bidens pilosa E Brachiaria plantaginea
}

\author{
Qualitative variables of spraying in common beans, Bidens pilosa and Brachiaria plantaginea
}

\author{
Andreia Cristina Peres Rodrigues ${ }^{1}$, Dagoberto Martins ${ }^{3}$, Neumarcio Vilanova da Costa ${ }^{2}$, \\ Leonildo Alves Cardoso ${ }^{3}$, Vanessa David Domingos ${ }^{3}$
}

\begin{abstract}
RESUMO
Conduziu-se este trabalho, com o objetivo de avaliar a qualidade da deposição da calda de pulverização em plantas de feijão (Phaseolus vulgaris L.), Bidens pilosa L. e de Brachiaria plantaginea (Link) Hitchc. presentes na linha e entrelinha da cultura. Foi utilizado como traçador o corante Azul Brilhante FDC-1 (500 ppm). Utilizaram-se cinco pontas de pulverização: jato plano (XR 110015 VS e XR 11002 VS), jato plano duplo (TJ60 11002 VS) e jato cônico (TX-6 VS e TX-8 VS) e dois volumes de aplicação 150 e $200 \mathrm{~L} \mathrm{ha}^{-1}$. O delineamento utilizado foi de blocos ao acaso, com 4 repetições. Foram amostradas 25 plantas em cada parcela/repetição, de plantas de feijão e plantas daninhas presentes na linha e na entrelinha da cultura. Após a aplicação, as plantas foram coletadas e lavadas em água destilada para quantificação do traçador em espectrofotômetro. Os dados ajustaram-se à curva de regressão pelo modelo de Gompertz. Os resultados evidenciaram que: para as plantas de feijão as pontas XR 110015 e TJ60 proporcionaram as deposições mais uniformes, nos volumes de 150 e $200 \mathrm{~L} \mathrm{ha}^{-1}$, respectivamente; a ponta TX-6 no volume de $150 \mathrm{~L} \mathrm{ha}^{-1}$ apresentou melhor uniformidade de distribuição para ambas as espécies de plantas daninhas presentes na linha da cultura; para as plantas daninhas presentes na entrelinha, no volume de $150 \mathrm{~L} \mathrm{ha}^{-1}$, destacaramse as pontas XR 110015 e TJ60 11002 para B. pilosa e B. plantaginea, respectivamente, no volume de $200 \mathrm{~L} \mathrm{ha}^{-1} \mathrm{destacou}^{-\mathrm{se}}$ a ponta TX-8 para ambas as espécies.
\end{abstract}

Termos para indexação: Phaseolus vulgaris, picão-preto, Bidens pilosa, capim-marmelada, Brachiaria plantaginea, bico, volume.

\section{ABSTRACT}

The objective of the present study was to evaluate the deposition quality of spray solution in common bean plants (Phaseolus vulgaris L.), Bidens pilosa L. and Brachiaria plantaginea (Link) Hitchc., present in the crop row and spacing row. Brilliant blue FDC - 1 was used as a tracer solution (500 ppm). Five nozzles were used: flat fan nozzle (XR 110015 VS and XR 11002 VS), double flat fan nozzle (TJ60 11002 VS) and cone nozzle (TX-6 VS and TX-8 VS), and two application volumes: 150 and 200 $\mathrm{L} \mathrm{ha}^{-1}$. A randomized blocks design was used, with four replications. 25 plants were sampled for replication in each plot, common beans as well as weeds in crop row and spacing row. After the application, the plants were collected and washed in distilled water for tracer quantification in spectrophotometer. The data adjusted to the regression curve according to the Gompertz model. The results showed that, for the common bean plants, XR 110015 and TJ60 nozzles had provided the most uniform depositions in 150 and 200 $\mathrm{L} \mathrm{ha}^{-1}$ application volume, respectively; the nozzle in the volume of $150 \mathrm{~L} \mathrm{ha}^{-1}$ had a more uniform distribution for both the species of harmful plants present in the crop line; for the weeds present in the crop row in a volume of $150 \mathrm{~L} \mathrm{ha}^{-1}$ the best performances were presented by the XR 110015 nozzle for $B$. pilosa and the TJ60 nozzle for B. plantaginea. For the volume of $200 \mathrm{~L} \mathrm{ha}^{-1}$, the TX-8 nozzle presented the best performance for both species.

Index terms: Common bean, Phaseolus vulgaris, hairy beggarticks, Bidens pilosa, alexandergrass, Brachiaria plantaginea, nozzle, volume.

(Recebido em 12 de julho de 2007 e aprovado em 10 de dezembro de 2009)

\section{INTRODUÇÃO}

Apesar da grande modernização das práticas culturais empregadas na cultura do feijão nos últimos anos, a produtividade atual ainda encontra-se muito aquém do potencial da cultura, que é superior a $4.500 \mathrm{~kg} \mathrm{ha}^{-1}$ (Cunha et al., 2005). Esse baixo rendimento é atribuído geralmente ao cultivo inadequado, condições climáticas adversas e incidência de pragas, doenças e plantas daninhas. Uma alternativa para contornar o problema quanto à presença de plantas daninhas é a aplicação de herbicidas, que diminuem a severidade da competição entre as plantas, podendo assim aumentar a produtividade da cultura. Dentre as plantas daninhas consideradas importantes competidoras na cultura do feijoeiro, são o picão-preto (Bidens pilosa L.) e o capim-marmelada [Brachiaria plantaginea (Link) Hitchc].

Vários estudos demonstram que a eficiência de herbicidas depende do volume de pulverização, da molécula ${ }^{1}$ Faculdade de Ciências Agronômicas/FCA/UNESP - Departamento de Produção Vegetal - Cx. P. 237 - 18603-970 - Botucatu, SP - andreia@fca.unesp.br
2Universidade Estadual do Oeste do Pararná/UNIOESTE Centro de Ciências Agrárias - Marechal Cândido Rondon, PR

${ }^{3}$ Faculdade de Ciências Agronômicas/FCA/UNESP - Departamento de Produção Vegetal - Botucatu, SP 
e do estádio de desenvolvimento das plantas daninhas (Foloni, 1995). Para avaliação do depósito do volume de pulverização há diversas metodologias: utilização de alvos artificiais (papel e lâminas de vidro), colocados próximos aos alvos naturais ou verdadeiros (folhas, caules, solo, etc.); papéis sensíveis, que mostram as marcas apenas em função da sensibilidade à umidade; utilização de corantes especiais, como fluorescentes (sensíveis à luz ultravioleta); uso da condutividade elétrica para determinação de concentrações de produtos fitossanitários, técnica esta que permite a utilização de alvo natural (Tomazela et al., 2006). Também existe a possibilidade de utilizar substâncias traçadoras, como corantes alimentícios e, entre estes, o Azul Brilhante FDC-1 avaliado por Palladini (2000).

A deposição e a uniformidade das aplicações dos produtos fitossanitários estão diretamente relacionadas com as características das plantas, tanto das folhas como da forma das plantas (Holloway, 1970), como características da própria pulverização (volume e tipo de ponta), por isso, estudos de tecnologias de aplicação tornaram-se importantes para melhorar a chegada da gotas de pulverização ao alvo (Costa, 1997).

A eficiência do tratamento fitossanitário não depende somente da quantidade de produto ativo depositado na planta, mas também da uniformidade da distribuição deste produto sobre a superfície alvo. Por esse motivo, é atribuída aos equipamentos de pulverização a função de distribuir o produto no tamanho de gotas adequado sobre a superfície alvo.

Conduziu-se este trabalho, com o objetivo de avaliar a qualidade da deposição da calda de pulverização em plantas de feijão, $B$. pilosa e de $B$. plantaginea presentes na linha e entrelinha da cultura, em aplicações de pósemergência, variando-se o volume de aplicação e o modelo de ponta de pulverização.

\section{MATERIAL E MÉTODOS}

O presente trabalho foi instalado e conduzido a campo, na Fazenda Experimental Lageado da Faculdade de Ciências Agronômicas - FCA/UNESP, campus de Botucatu/SP no ano de 2005. Utilizou-se para o estudo a cultivar carioca de feijão, bem como as plantas daninhas $B$. pilosa e a $B$. plantaginea que são espécies comumente encontradas na cultura.

A semeadura do feijão foi realizada no dia 02/09/2005, no espaçamento de $0,5 \mathrm{~m}$ entre as linhas na densidade de 11-12 sementes $\mathrm{m}^{-1}$. As plantas de $B$. pilosa surgiram naturalmente na área do experimento sem a necessidade de serem semeadas. Sementes de B. plantaginea foram semeadas no mesmo dia, a lanço, em todas as parcelas.
O estudo foi conduzido no delineamento em blocos ao acaso, com 4 repetições, na qual se avaliaram pontas de pulverização: jato plano (XR 110015 VS e XR 11002 VS), jato plano duplo (TJ60 11002 VS) e jato cônico (TX-6 VS e TX-8 VS); e dois volumes de pulverização (150 e $200 \mathrm{~L} \mathrm{ha}^{-1}$ ).

Para a pulverização do traçante foi preparada uma solução do corante Azul Brilhante FDC-1 numa concentração de 500 ppm. A aplicação da calda foi realizada no dia 01/10/2005, com o auxílio de um pulverizador costal, pressurizado a $\mathrm{CO}_{2}$, com pressão de $175 \mathrm{kPa}$, com a velocidade ajustada dependendo do tipo de ponta e do volume utilizado. Durante a pulverização, a temperatura e a umidade relativa do ar variaram de $28,9^{\circ} \mathrm{C}$ a $33,9^{\circ} \mathrm{C}$ e $33 \%$ a $65 \%$, respectivamente, sendo que o vento encontravase na forma de rajadas a $2,5 \mathrm{~km} \mathrm{~h}^{-1} \mathrm{em}$ média.

Na ocasião da aplicação da calda de pulverização, as plantas de feijão encontravam-se no estádio V4 com três folhas verdadeiras, já as plantas daninhas apresentavam de 2 a 3 pares de folhas para B. pilosa e 3 a 4 perfilhos para $B$. plantaginea.

As amostras foram coletadas após a pulverização, armazenadas em sacos plásticos e levadas ao laboratório onde foram lavadas com água destilada $(100 \mathrm{~mL})$ para retirada do corante. Em cada parcela, foram coletadas 25 plantas daninhas na linha e entrelinha e 25 plantas de feijão. Cada planta, selecionada ao acaso, dentro da faixa de aplicação da barra foi considerada uma repetição, totalizando 100 repetições. Após a lavagem, as plantas foram colocadas em sacos de papel etiquetados e levadas à uma estufa de ventilação forçada de ar para secagem. Após 72 horas, a uma temperatura de $60^{\circ} \mathrm{C}$, as plantas foram pesadas determinando-se a massa seca.

Determinou-se a quantidade de traçante depositada utilizando-se um espectofotômetro, cujos resultados da leitura em absorbância no comprimento de onda de $630 \mathrm{~nm}$ proporcionaram a transformação em $\mathrm{mg} \mathrm{L}^{-1}$, de acordo com o coeficiente angular da curva padrão. Os valores de depósitos foram posteriormente transformados em $\mathrm{mL} \mathrm{cm}^{-2}$ e mL planta $^{-1}$, conforme metodologia descrita por Palladini (2000).

Os dados obtidos dos depósitos em porcentagem foram ajustados pelo modelo de Gompertz $\left(F=e^{\wedge}\left(a-e^{\wedge}(-b-\right.\right.$ $\left.\mathrm{c}^{*} \mathrm{X}\right)$ )), para representar a frequência acumulada $(\mathrm{F}) \mathrm{da}$ deposição da calda pulverizada em percentagem. Adotouse o valor 4,60517 para o paramentro "a" do modelo, o qual representa a assíntota máxima da curva, em que "e $\mathrm{e}$ " $=100$, conforme o modelo ajustado por Velini (1995). O deslocamento da curva ao longo do eixo x é representado pelo módulo do parâmetro "a", e a inclinação ou concavidade da curva em relação a frequência acumulada, pelo parâmetro "c". Além 
disso, para melhor visualização, optou-se por apresentar as frequências não-acumuladas ( $\mathrm{FN}=\mathrm{c}^{*} \mathrm{e}^{\wedge}\left(\mathrm{a}-\mathrm{b}-\mathrm{c} * \mathrm{X}-\mathrm{e}^{\wedge}(-\mathrm{b}-\right.$ $\left.\left.\mathrm{c}^{*} \mathrm{X}\right)\right)$ ), que correspondem a derivada da primeira do modelo. As frequências acumuladas e não-acumuladas do modelo de Gompertz permitem a interpretação biológica dos resultados. Para facilitar a visualização das informações, a derivada primeira corresponde à moda da deposição da solução pulverizada nos alvos específicos (Velini, 1995).

Os valores de média, moda e mediana também foram estabelecidos no trabalho. A precisão do ajuste dos dados do modelo de Gompertz foi avaliada por meio dos coeficientes de determinação $\left(\mathrm{R}^{2}\right)$ e pela soma dos quadrados dos resíduos das equações.

\section{RESULTADOS E DISCUSSÃO}

A qualidade da deposição pode ser analisada por meio dos gráficos correspondentes aos alvos, que foram ajustados pelo modelo matemático de Gompertz e mostram frequência acumulada (FA\%), ou seja, os valores medianos para as diferentes porcentagens de população de planta, e a freqüência não-acumulada (FNA\%), sendo que o pico da curva fornece a moda ou seja, os valores de depósitos que aparecem com maior frequência, e a concavidade da curva mostra a uniformidade de deposição. Em relação à concavidade, quanto mais plana a curva, maior é a frequência dos valores extremos (Velini, 1995), caracterizando maior amplitude de deposito na população amostrada, ou seja, maiores diferenças entre os maiores e menores volumes depositados nas plantas.

Observa-se, quanto aos depósitos nas plantas de feijão, que a ponta TJ 60 proporcionou maior valor de moda, o que corresponde ao maior depósito e a maior regularidade na distribuição da calda de pulverização sobre os alvos, utilizando-se o volume de $150 \mathrm{~L} \mathrm{ha}^{-1}$, seguido das pontas XR 110015 e TX-6, sendo que estes dados corroboram os de Maciel et al. (2001) que constataram maiores depósitos em plantas de feijão, proporcionados pela ponta XR 11002 (jato plano), em relação à ponta TX-4 (jato cônico) no volume de aplicação de $100 \mathrm{~L} \mathrm{ha}^{-1}$. Contudo, no volume de $200 \mathrm{~L} \mathrm{ha}^{-1}$, a melhor deposição e uniformidade das gotas de pulverização foi conferida pela ponta XR 11002, seguida das pontas TX-8 e TJ60. Isso pode ser explicado pela diferença na inclinação das curvas representadas pela Figuras 1C e 1D, comparandose o comportamento de pontas iguais submetidas a diferentes volumes ou, ainda, por meio do parâmetro "c" do modelo de Gompertz (Tabela 1), em que os maiores valores indicam menores inclinações das curvas e assim maior uniformidade de deposição da calda de pulverização. Ressalta-se que os resultados conferidos para o volume de $200 \mathrm{~L} \mathrm{ha}^{-1}$ também corroboram os de Maciel et al. (2001).

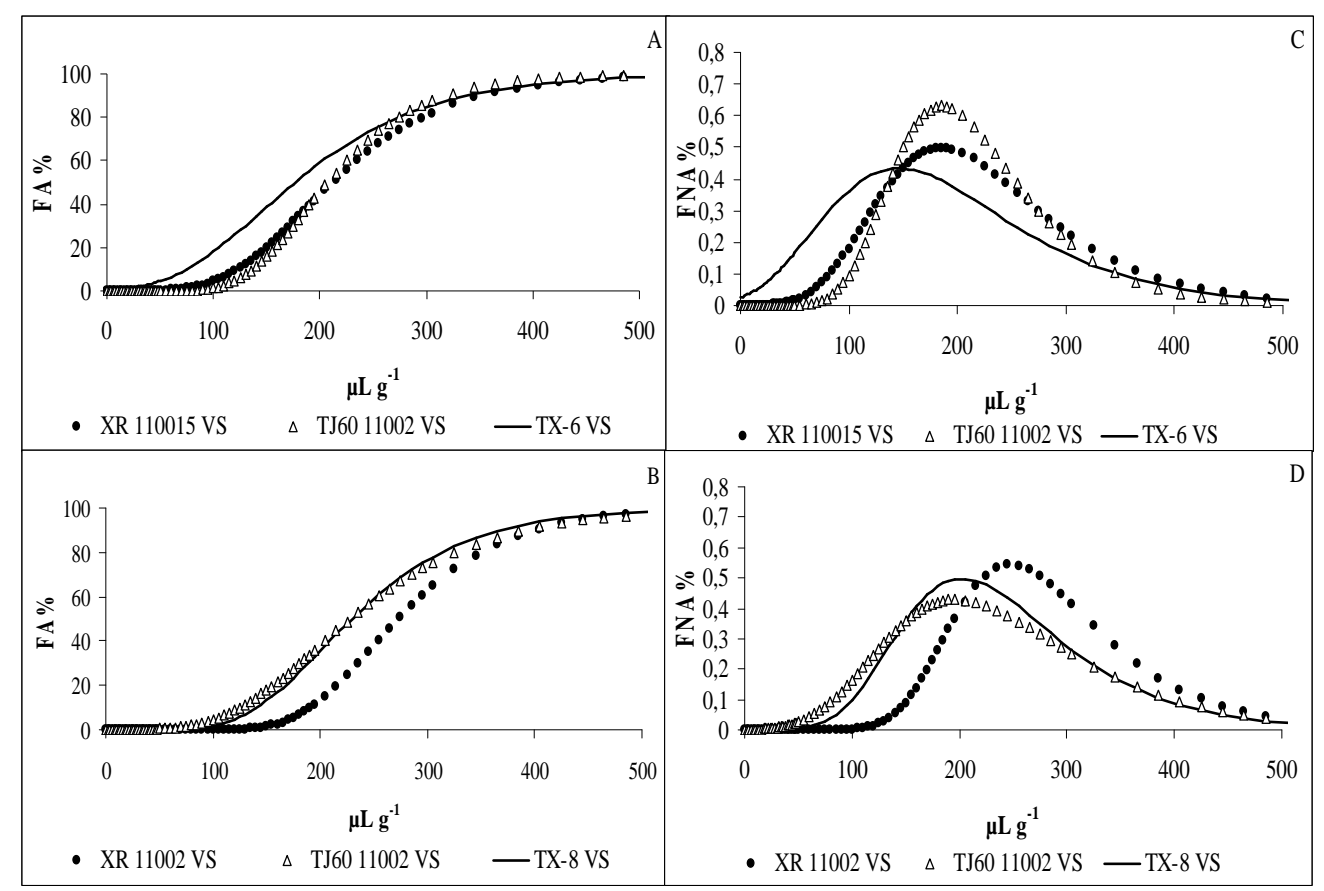

Figura 1 - Frequência acumuladas (FA\%): (A-B), e não-acumuladas (FNA\%): (C-D), em função da deposição da calda de pulverização em $\mu \mathrm{L} \mathrm{g}^{-1}$ de massa seca de plantas de feijão. (A-C) volume de aplicação de $150 \mathrm{~L} \mathrm{ha}^{-1} \mathrm{e}(\mathrm{B}-\mathrm{D})$ volume de aplicação $200 \mathrm{~L} \mathrm{ha}^{-1}$. 
A uniformidade da deposição da calda de pulverização destaca-se como fator importante no desempenho dos produtos fitossanitários, principalmente quando se considera produtos de ação de contato que exigem maior área de cobertura do alvo.

Observa-se, também que, com o aumento do volume aplicado ocorreu um acréscimo na quantidade do volume de pulverização depositado nas plantas de feijão, independente da ponta de pulverização.

Na Tabela 2 e Figura 2, de forma similar aos resultados das plantas de feijão, a ponta TJ60 proporcionou os maiores depósitos de calda nas plantas de $B$. pilosa encontrados na linha da cultura do feijão com o volume de aplicação de 150 $\mathrm{L} \mathrm{ha}^{-1}$, sendo superior somente em $4 \%$ à ponta XR 110015 , entretanto, a ponta TX-6, apresentou deposição bem reduzida em relação às outras pontas (TJ60 e XR 110015) na ordem de 91,3 e 90,9\%, respectivamente (Tabela 2). Analisando o Volume de $200 \mathrm{~L} \mathrm{ha}^{-1}$, ocorreu o inverso, sendo que o maior depósito encontrado nas plantas de $B$. pilosa foi proporcionado pela ponta $\mathrm{TX}-8$, que apresentou deposições superiores em 37,4 e 48,8\% em relação às pontas XR 11002 e TJ60, respectivamente. Analisando a uniformidade de distribuição da calda de pulverização, verifica-se que a ponta TX-6 no volume de $150 \mathrm{~L} \mathrm{ha}^{-1}$ foi a que apresentou maior uniformidade de deposição em relação as demais, que evidenciaram um comportamento semelhante, sendo desuniformes na distribuição das gotas (Figura 2C). Já, a ponta XR 11002 destacou-se com a melhor uniformidade de distribuição, seguida da ponta TX-8 no volume de $200 \mathrm{~L}$ ha $^{-1}$ (Figura 2D).
Registra-se, ainda, que a ponta TX-6 VS, apesar de ter proporcionado a maior uniformidade de deposição (menores inclinações nas curvas e/ou maiores valores de "c"), proporcionou a menor quantidade de depósitos nas plantas, demonstrando que nem sempre a ponta de que deposita maior quantidade de calda é aquela que apresenta melhor uniformidade de distribuição das gotas.

Costa (1997), concluiu que a irregularidade na uniformidade de distribuição das gotas pode levar à necessidade de aumento da dose aplicada e que a proximidade das plantas daninhas com a cultura desuniformiza e reduz os depósitos da calda de aplicação.

A ponta TX-6, no volume de $150 \mathrm{~L} \mathrm{ha}^{-1}$, foi a que proporcionou o melhor depósito nas plantas de $B$. pilosa encontradas na entrelinha da cultura do feijão, quando comparadas aos outros tratamentos (Tabela 3). Esse resultado corrobora os de Martins (2004), quando verificou na cultura da batata que o uso de volumes maiores de calda de pulverização, não determinavam, necessariamente, em maiores depósitos.

A ponta TX-6 proporcionou deposição superior, da ordem de 38,1 e 55,4\% em relação às pontas TJ60 e XR 110015 , respectivamente. Observa-se que no volume de $200 \mathrm{~L} \mathrm{ha}^{-1}$ ocorreu o inverso, pois a ponta que proporcionou maior depósito foi a ponta XR 11002, com valores de 56,5 e $62,7 \%$ superior para as pontas TX-8 e TJ60, respectivamente. Ressalta-se, ainda, que a ponta XR 110015 foi a que proporcionou melhor uniformidade de distribuição no volume de $150 \mathrm{~L} \mathrm{ha}^{-1}$, as demais pontas apresentaram deposição mais desuniforme (Figura 3C).

Tabela 1 - Resultados das análises de regressão entre as freqüências acumuladas de depósito da solução pulverizada, utilizando o modelo de Gompertz, médias, modas e medianas da deposição em plantas de feijão, em diferentes pontas de pulverização e volumes de aplicação. Botucatu-SP, 2005.

\begin{tabular}{|c|c|c|c|c|c|c|c|}
\hline \multirow{3}{*}{ Modelo } & & \multicolumn{6}{|c|}{ Frequência Acumulada $(\%)=\mathrm{e}^{\wedge}\left[\mathrm{A}-\mathrm{e}^{\wedge}(-\mathrm{B}-\mathrm{C} * \mathrm{X})\right]$} \\
\hline & & XR 110015 & TJ60 11002 & TX-6 & XR 11002 & TJ60 11002 & TX-8 \\
\hline & & & $150 \mathrm{Lha}^{-1}$ & & & $200 \mathrm{Lha}^{-1}$ & \\
\hline \multirow{3}{*}{$\begin{array}{l}\text { Estimativa } \\
\text { dos } \\
\text { parâmetros }\end{array}$} & A & 4,60517 & 4,60517 & 4,60517 & 4,60517 & 4,60517 & 4,60517 \\
\hline & $\mathrm{B}$ & $-2,50348$ & $-3,18505$ & $-1,71566$ & $-3,65996$ & $-2,29456$ & $-2,73616$ \\
\hline & $\mathrm{C}$ & 0,01351 & 0,01714 & 0,01177 & 0,01475 & 0,01166 & 0,01351 \\
\hline \multicolumn{2}{|l|}{$\mathrm{F}_{\text {Regressão }}$} & $16774,390 * *$ & $12689,832 * *$ & $832,221 * *$ & $10751,594 * *$ & $16063,979 * *$ & $12073,973 * *$ \\
\hline \multicolumn{2}{|l|}{$\mathrm{R}^{2}$} & 0,988 & 0,984 & 0,774 & 0,982 & 0,988 & 0,984 \\
\hline \multicolumn{2}{|l|}{ Média } & 230,64 & 224,32 & 181,44 & 286,83 & 243,93 & 243,55 \\
\hline \multicolumn{2}{|l|}{ Moda } & 185,31 & 185,78 & 145,82 & 248,08 & 196,73 & 202,47 \\
\hline \multicolumn{2}{|l|}{ Mediana } & 212,44 & 207,16 & 176,97 & 272,92 & 228,16 & 229,59 \\
\hline
\end{tabular}

$\mathrm{X}=$ porcentagem de depósito do traçador em relação ao total aplicado; A, B e C são estimativas dos parâmetros do modelo para o traçador.

** significativo a $1 \%$ de probabilidade. 
No volume de $200 \mathrm{~L} \mathrm{ha}^{-1}$, as pontas XR 11002 VS e TX-8 VS apresentaram uniformidade semelhante, enquanto a ponta TJ60 teve um pico mais plano em relação às demais pontas, indicando uma maior desuniformidade (Figura 3D).

Verifica-se, no volume de $150 \mathrm{~L} \mathrm{ha}^{-1}$, que a ponta TJ60 11002 VS proporcionou um maior depósito de calda de pulverização nas plantas de $B$. plantaginea encontrados na linha da cultura do feijão, seguida pela ponta XR 110015 (Tabela 4). A ponta TX-6 teve uma deposição bastante reduzida em relação às outras pontas, sendo que esta apresentou 98,2 e 97,7\% de decréscimos nos depósitos em relação às pontas TJ60 e XR 110015, respectivamente. No volume de $200 \mathrm{~L} \mathrm{ha}^{-1}$, a ponta TX-8 proporcionou maior deposição, seguida pelas pontas XR 11002 VS e TJ60, que apresentaram depósitos superiores em 3,5 e 46,5\%, respectivamente. Entretanto, as grandes discrepâncias encontradas entre as maiores e as menores quantidades médias de calda de pulverização em função das diferentes pontas e volumes de aplicação, apresentaram expressivas alterações. As diferenças entre os maiores e os menores depósitos para o volume de 150, em comparação a 200 $\mathrm{L} \mathrm{ha}^{-1}$, no mesmo modelo de ponta, foram de 151,91; 158,54 e 353,28 em $\mu \mathrm{L} \mathrm{g}^{-1}$ de massa seca para os modelos de ponta XR; TJ60 e TX, respectivamente.

Verifica-se que a melhor uniformidade distribuição nas plantas de $B$. plantaginea encontradas na linha da cultura do feijão ocorreu com ponta TX-6, utilizando-se o volume de $150 \mathrm{~L} \mathrm{ha}^{-1}$, mesmo sendo esta ponta a que apresentou os menores depósitos (Figura 4C). Em relação às outras pontas testadas verifica-se que o pico de deposição foi bastante plano, representando grande desuniformidade na deposição. Da mesma forma, observase que as pontas utilizadas no volume de $200 \mathrm{~L} \mathrm{ha}^{-1}$ apresentaram-se bastante desuniforme (Figura 4D).

Para os valores de deposição verificados nas plantas de $B$. plantaginea na entrelinha da cultura, a ponta XR 110015 proporcionou o melhor deposito nas plantas no volume de $150 \mathrm{~L} \mathrm{ha}^{-1}$, seguida pelas pontas TX-6 e TJ60, com valor de moda superior e da ordem de 4,8 e 60,4\%, respectivamente (Tabela 5 e Figura 5). Para o volume de $200 \mathrm{~L} \mathrm{ha}^{-1}$ ao contrário do que ocorreu com volume menor, a ponta TJ60 proporcionou maior depósito, sendo 61,3 e $67,6 \%$ superior às pontas XR 11002 e TX-8, respectivamente.

A redução do volume proporcionou incrementos nos depósitos para as pontas XR e TX, corroborando os resultados encontrados por Tomazela et al. (2006) que, ao utilizar a ponta XR 8001EVS, verificaram que a diminuição do volume de calda de pulverização promoveu incrementos significativos na porcentagem de depósitos nas plantas de B. plantaginea.

Quanto à uniformidade de distribuição da calda de pulverização no volume de $150 \mathrm{~L} \mathrm{ha}^{-1}$, a ponta TJ60 proporcionou maior uniformidade, enquanto que as pontas XR 110015 e TX-6, apresentaram comportamento semelhante (Figura 5C). No volume de $200 \mathrm{~L} \mathrm{ha}^{-1}$, a ponta que proporcionou melhor uniformidade foi a TX-8, enquanto que as demais pontas utilizadas, apresentaram depósitos bem desuniformes (Figura 5D).

Tabela 2 - Resultados das análises de regressão entre as freqüências acumuladas de depósito da solução pulverizada, utilizando o modelo de Gompertz, médias, modas e medianas da deposição em B. pilosa na linha da cultura do feijão, em diferentes pontas de pulverização e volumes de aplicação. Botucatu-SP, 2005.

\begin{tabular}{|c|c|c|c|c|c|c|c|}
\hline \multirow{3}{*}{ Modelo } & & \multicolumn{6}{|c|}{ Frequência Acumulada $(\%)=\mathrm{e}^{\wedge}\left[\mathrm{A}-\mathrm{e}^{\wedge}\left(-\mathrm{B}-\mathrm{C}^{*} \mathrm{X}\right)\right]$} \\
\hline & & XR 110015 & TJ60 11002 & TX-6 & XR 11002 & TJ60 11002 & TX-8 \\
\hline & & & $150 \mathrm{Lha}^{-1}$ & & & $200 \mathrm{Lha}^{-1}$ & \\
\hline \multirow{3}{*}{$\begin{array}{l}\text { Estimativa } \\
\text { dos } \\
\text { parâmetros }\end{array}$} & A & 4,60517 & 4,60517 & 4,60517 & 4,60517 & 4,60517 & 4,60517 \\
\hline & $\mathrm{B}$ & $-1,07162$ & $-1,14141$ & $-0,19961$ & $-1,57086$ & $-0,96266$ & $-2,36717$ \\
\hline & $\mathrm{C}$ & 0,00701 & 0,00717 & 0,01439 & 0,00806 & 0,00536 & 0,00761 \\
\hline \multicolumn{2}{|l|}{$\mathrm{F}_{\text {Regressão }}$} & $13879,064 * *$ & $21909,944 * *$ & $1014,244 * *$ & $5214,150 * *$ & $24980,923^{* *}$ & $9290,776 * *$ \\
\hline \multicolumn{2}{|l|}{$\mathrm{R}^{2}$} & 0,986 & 0,991 & 0,813 & 0,962 & 0,992 & 0,979 \\
\hline \multicolumn{2}{|l|}{ Média } & 272,73 & 251,53 & 69,28 & 262,25 & 364,48 & 575,88 \\
\hline \multicolumn{2}{|l|}{ Moda } & 152,84 & 159,29 & 13,87 & 194,85 & 179,64 & 311,24 \\
\hline \multicolumn{2}{|l|}{ Mediana } & 205,12 & 210,44 & 39,34 & 240,31 & 248,03 & 359,43 \\
\hline
\end{tabular}

$\mathrm{X}=$ porcentagem de depósito do traçador em relação ao total aplicado; A, B e C são estimativas dos parâmetros do modelo para o traçador.

** significativo a $1 \%$ de probabilidade. 


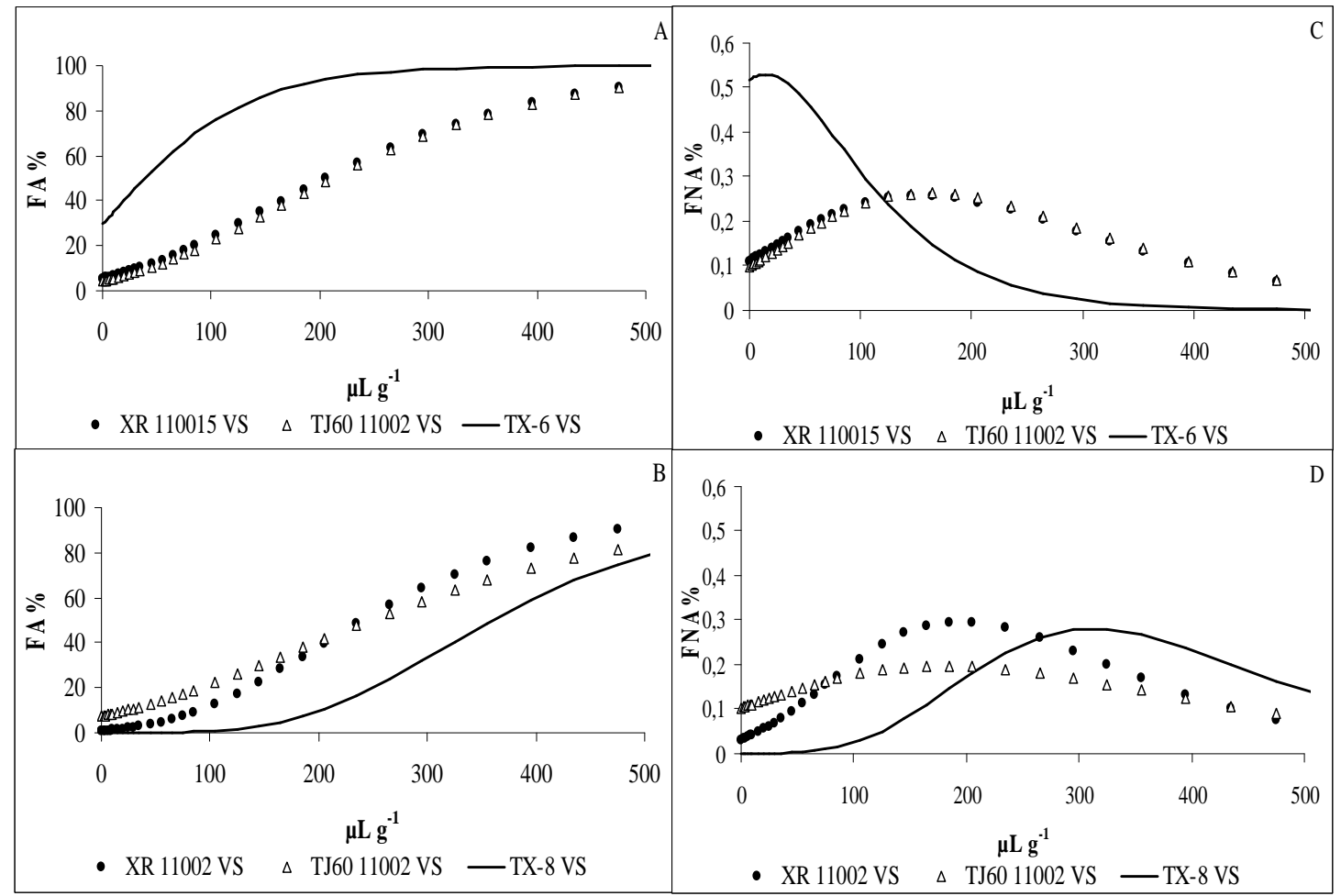

Figura 2 - Frequência acumuladas (FA\%): (A-B), e não-acumuladas (FNA\%): (C-D), em função da deposição da calda de pulverização em $\mu \mathrm{L} \mathrm{g}^{-1}$ de massa seca de plantas de B. pilosa, na linha da cultura do feijão. (A-C) volume de aplicação de $150 \mathrm{~L} \mathrm{ha}^{-1}$ e (B-D) volume de aplicação $200 \mathrm{~L} \mathrm{ha}^{-1}$.

Tabela 3 - Resultados das análises de regressão entre as frequências acumuladas de depósito da solução pulverizada, utilizando o modelo de Gompertz, médias, modas e medianas da deposição em B. pilosa na entrelinha da cultura do feijão, em diferentes pontas de pulverização e volumes de aplicação. Botucatu-SP, 2005.

\begin{tabular}{|c|c|c|c|c|c|c|c|}
\hline \multirow{3}{*}{ Modelo } & & \multicolumn{6}{|c|}{ Frequência Acumulada $(\%)=\mathrm{e}^{\wedge}\left[\mathrm{A}-\mathrm{e}^{\wedge}\left(-\mathrm{B}-\mathrm{C}^{*} \mathrm{X}\right)\right]$} \\
\hline & & XR 110015 & TJ60 11002 & TX-6 & XR 11002 & TJ60 11002 & TX-8 \\
\hline & & & $150 \mathrm{Lha}^{-1}$ & & & $200 \mathrm{Lha}^{-1}$ & \\
\hline \multirow{3}{*}{$\begin{array}{l}\text { Estimativa } \\
\text { dos } \\
\text { parâmetros }\end{array}$} & A & 4,60517 & 4,60517 & 4,60517 & 4,60517 & 4,60517 & 4,60517 \\
\hline & $\mathrm{B}$ & $-1,00846$ & $-0,92813$ & $-1,51022$ & $-1,73413$ & $-0,58041$ & $-0,79223$ \\
\hline & $\mathrm{C}$ & 0,00703 & 0,00466 & 0,00469 & 0,00635 & 0,00570 & 0,00666 \\
\hline \multicolumn{2}{|l|}{$\overline{F_{\text {Regressão }}}$} & $35956,942 * *$ & $17996,545^{* *}$ & $37983,836 * *$ & $14635,552 * *$ & $6695,143^{* *}$ & $22051,050 * *$ \\
\hline \multicolumn{2}{|l|}{$\mathrm{R}^{2}$} & 0,994 & 0,989 & 0,995 & 0,986 & 0,970 & 0,991 \\
\hline \multicolumn{2}{|l|}{ Média } & 231,11 & 360,25 & 500,25 & 578,25 & 221,76 & 216,52 \\
\hline \multicolumn{2}{|l|}{ Moda } & 143,52 & 199,28 & 322,10 & 273,12 & 101,83 & 118,87 \\
\hline \multicolumn{2}{|l|}{ Mediana } & 195,68 & 277,97 & 400,28 & 330,84 & 166,13 & 173,86 \\
\hline
\end{tabular}

$\mathrm{X}=$ porcentagem de depósito do traçador em relação ao total aplicado; A, B e C são estimativas dos parâmetros do modelo para o traçador.

** significativo a $1 \%$ de probabilidade. 


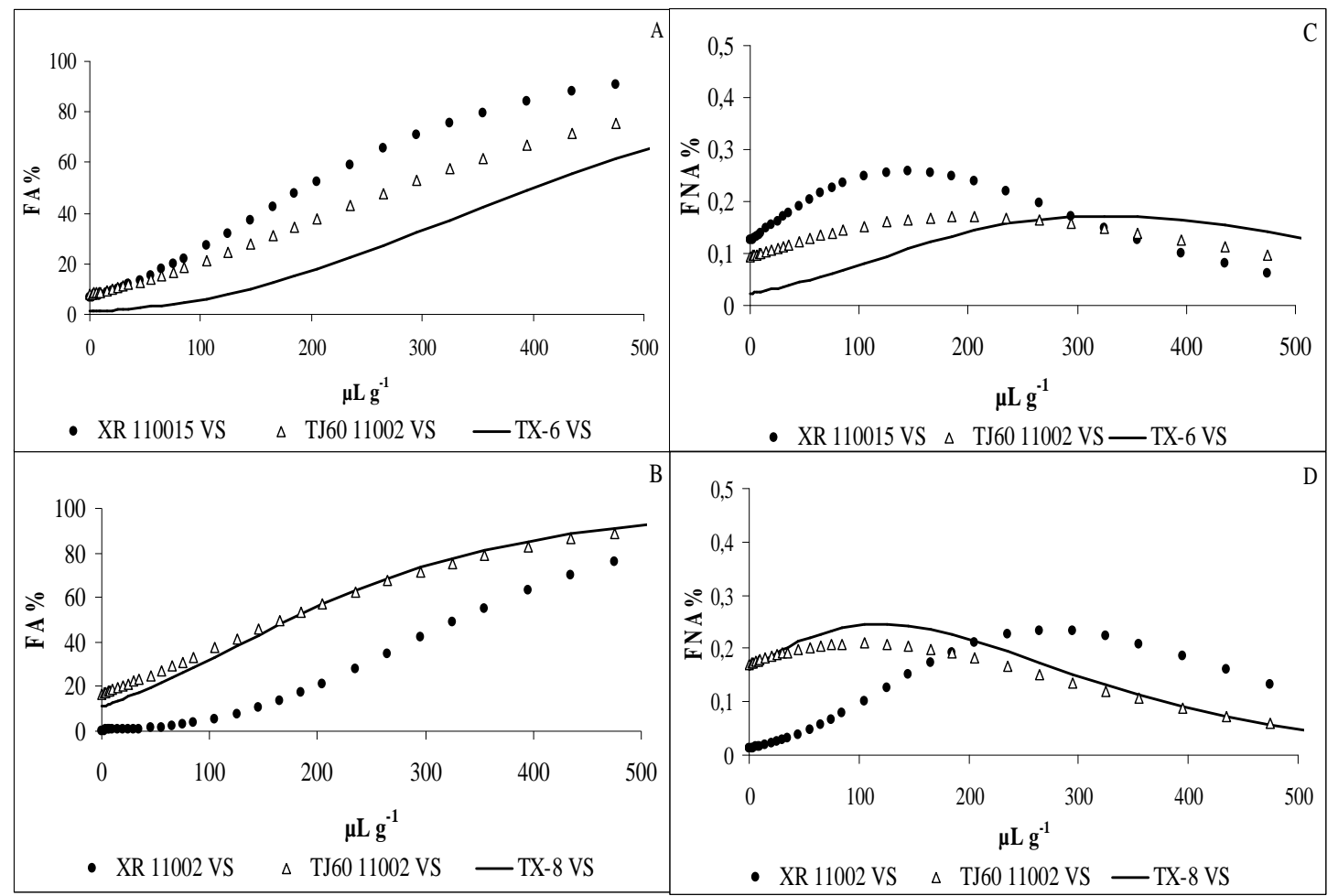

Figura 3 - Frequência acumuladas (FA\%): (A-B), e não-acumuladas (FNA\%): (C-D), em função da deposição da calda de pulverização em $\mu \mathrm{L} \mathrm{g}^{-1}$ de massa seca de plantas de B. pilosa, na entrelinha da cultura do feijão. (A-C) volume de aplicação de $150 \mathrm{~L} \mathrm{ha}^{-1}$ e (B-D) volume de aplicação $200 \mathrm{~L} \mathrm{ha}^{-1}$.

Tabela 4 - Resultados das análises de regressão entre as frequências acumuladas de depósito da solução pulverizada, utilizando o modelo de Gompertz, médias, modas e medianas da deposição em B. plantaginea na linha da cultura do feijão, em diferentes pontas de pulverização e volumes de aplicação. Botucatu-SP, 2005.

\begin{tabular}{lccccccc}
\hline \multirow{2}{*}{ Modelo } & \multicolumn{6}{c}{ Frequência Acumulada $(\%)=\mathrm{e}^{\wedge}\left[\mathrm{A}-\mathrm{e}^{\wedge}\left(-\mathrm{B}-\mathrm{C}^{*} \mathrm{X}\right)\right]$} \\
\cline { 2 - 8 } & \cline { 2 - 7 } & XR 110015 & TJ60 11002 & TX-6 & XR 11002 & TJ60 11002 & TX-8 \\
\cline { 2 - 8 } \multicolumn{1}{c}{ Estimativa } & $\mathrm{A}$ & 4,60517 & 4,60517 & 4,60517 & 4,60517 & 4,60517 & 4,60517 \\
parâmetros & $\mathrm{B}$ & $-1,32988$ & $-1,19155$ & $-0,15391$ & $-0,72945$ & $-0,91103$ & $-1,67621$ \\
\hline $\mathrm{F}_{\text {Regressão }}$ & $\mathrm{C}$ & 0,00668 & 0,00454 & 0,03295 & 0,00367 & 0,00626 & 0,00616 \\
$\mathrm{R}^{2}$ & $16774,390^{* *}$ & $12689,832^{* *}$ & $832,221^{* *}$ & $10751,594^{* *}$ & $16063,979^{* * *}$ & $12073,973^{* *}$ \\
\hline Média & 0,988 & 0,984 & 0,774 & 0,982 & 0,988 & 0,984 \\
Moda & 331,78 & 465,49 & 44,23 & 483,69 & 306,95 & 397,51 \\
Mediana & 199,08 & 262,70 & 4,67 & 198,78 & 145,64 & 272,29 \\
\hline X & 253,94 & 343,50 & 15,80 & 298,66 & 204,23 & 331,83 \\
\hline
\end{tabular}

$\mathrm{X}=$ porcentagem de depósito do traçador em relação ao total aplicado; A, B e C são estimativas dos parâmetros do modelo para o traçador.

** significativo a $1 \%$ de probabilidade. 


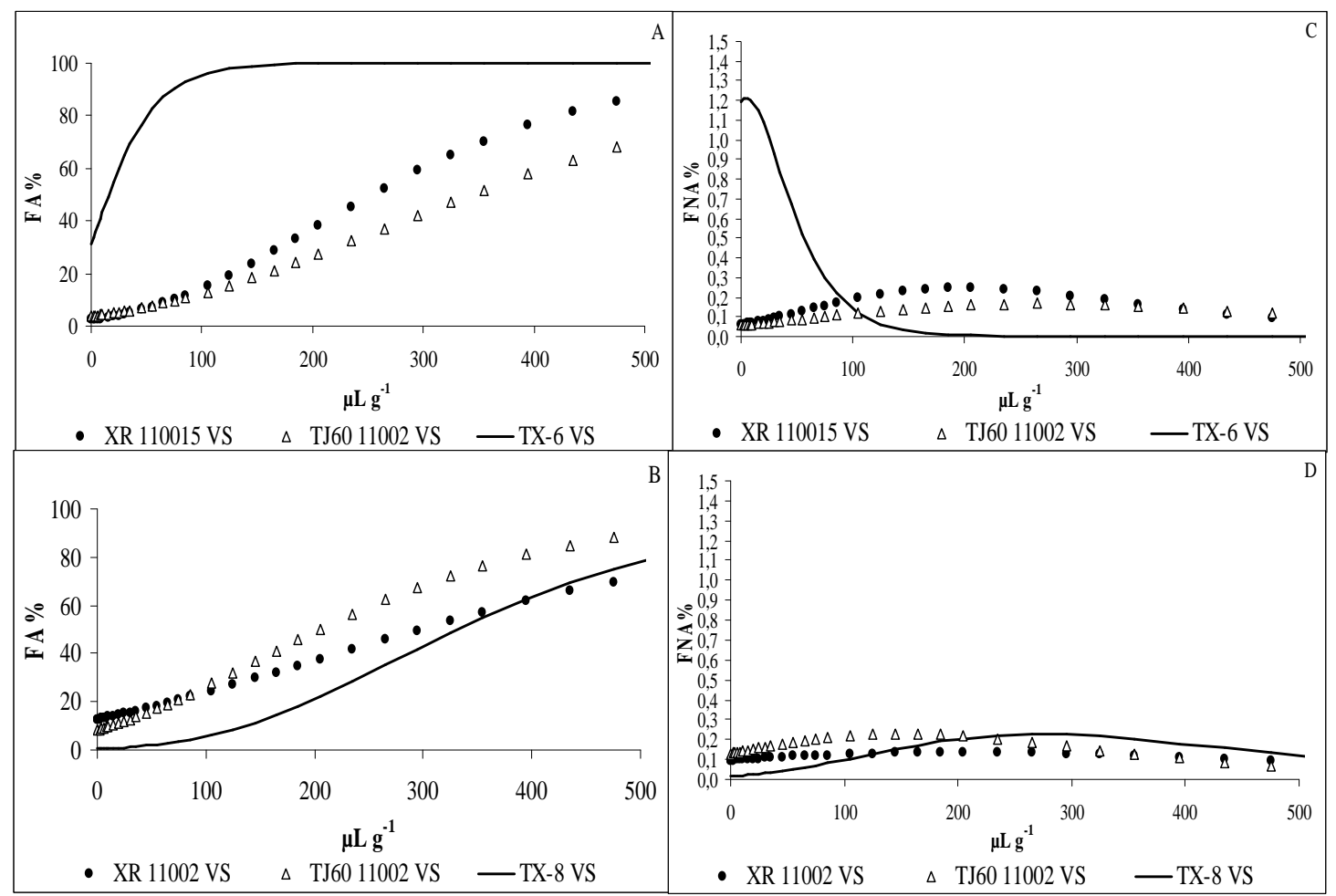

Figura 4 - Frequência acumuladas (FA\%): (A-B), e não-acumuladas (FNA\%): (C-D), em função da deposição da calda de pulverização em $\mu \mathrm{L} \mathrm{g}^{-1}$ de massa seca de plantas de B. plantaginea, na linha da cultura do feijão. (A-C) volume de aplicação de $150 \mathrm{~L} \mathrm{ha}^{-1}$ e (B-D) volume de aplicação $200 \mathrm{~L} \mathrm{ha}^{-1}$.

Tabela 5 - Resultados das análises de regressão entre as frequências acumuladas de depósito da solução pulverizada, utilizando o modelo de Gompertz, médias, modas e medianas da deposição em B. plantaginea na entrelinha da cultura do feijão, em diferentes pontas de pulverização e volumes de aplicação. Botucatu-SP, 2005.

\begin{tabular}{|c|c|c|c|c|c|c|c|}
\hline \multirow{3}{*}{ Modelo } & & \multicolumn{6}{|c|}{ Frequência Acumulada $(\%)=\mathrm{e}^{\wedge}\left[\mathrm{A}-\mathrm{e}^{\wedge}\left(-\mathrm{B}-\mathrm{C}^{*} \mathrm{X}\right)\right]$} \\
\hline & & XR 110015 & TJ60 11002 & TX-6 & XR 11002 & TJ60 11002 & TX-8 \\
\hline & & & $150 \mathrm{Lha}^{-1}$ & & & 200 Lha $^{-1}$ & \\
\hline \multirow{3}{*}{$\begin{array}{l}\text { Estimativa } \\
\text { dos } \\
\text { parâmetros }\end{array}$} & A & 4,60517 & 4,60517 & 4,60517 & 4,60517 & 4,60517 & 4,60517 \\
\hline & $\mathrm{B}$ & $-1,41402$ & $-0,67857$ & $-1,29054$ & $-0,75521$ & $-2,02208$ & $-1,14401$ \\
\hline & $\mathrm{C}$ & 0,00747 & 0,00906 & 0,00716 & 0,00529 & 0,00549 & 0,00957 \\
\hline \multicolumn{2}{|l|}{ Fegressão } & $15478,822 * *$ & $30375,860 * *$ & $13022,145 * *$ & $7715,791 * *$ & $10080,439 * *$ & $30375,860 * *$ \\
\hline \multicolumn{2}{|l|}{$\mathrm{R}^{2}$} & 0,987 & 0,993 & 0,985 & 0,974 & 0,980 & 0,993 \\
\hline \multicolumn{2}{|l|}{ Média } & 306,49 & 159,62 & 308,24 & 658,88 & 568,70 & 184,56 \\
\hline \multicolumn{2}{|l|}{ Moda } & 189,32 & 74,93 & 180,24 & 142,76 & 368,60 & 119,53 \\
\hline \multicolumn{2}{|l|}{ Mediana } & 238,39 & 115,40 & 231,42 & 212,05 & 435,42 & 157,83 \\
\hline
\end{tabular}

$\mathrm{X}=$ porcentagem de depósito do traçador em relação ao total aplicado; A, B e C são estimativas dos parâmetros do modelo para o traçador.

** significativo a $1 \%$ de probabilidade. 


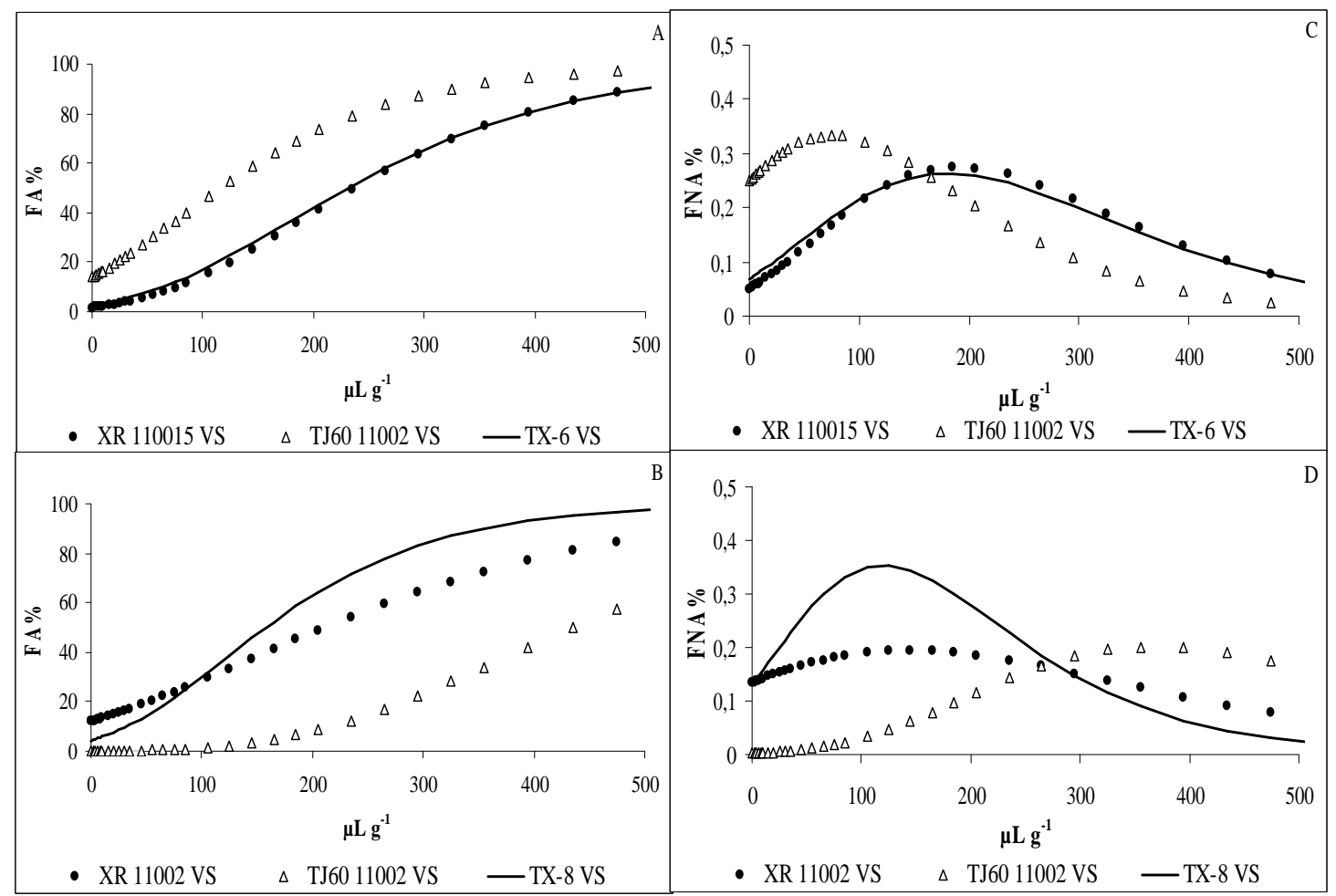

Figura 5 - Frequência acumuladas (FA\%): (A-B), e não-acumuladas (FNA\%): (C-D), em função da deposição da calda de pulverização em $\mu \mathrm{L} \mathrm{g}^{-1}$ de massa seca de plantas de B. plantaginea, na entrelinha da cultura do feijão. (A-C) volume de aplicação de $150 \mathrm{~L} \mathrm{ha}^{-1}$ e (B-D) volume de aplicação $200 \mathrm{~L} \mathrm{ha}^{-1}$.

A ponta TJ60, no volume de $150 \mathrm{~L} \mathrm{ha}^{-1}$, bem como a ponta TX-8, no volume de $200 \mathrm{~L} \mathrm{ha}^{-1}$, foram as que apresentaram os menores valores de deposição, contudo, foram as que proporcionaram os melhores resultados de uniformidade de distribuição da calda de pulverização em ambos os volumes (Figura 5). Segundo Negrisoli et al. (2002), as irregularidades dos depósitos podem levar a necessidade de aumento nas doses aplicadas, acarretando em incrementos das doses necessárias para compensar as perdas. Apesar disso, a pesquisa ressalta que são escassos os estudos que procuram estabelecer a dispersão de depósitos no solo ou em populações de plantas daninhas.

Souza et al. (2007), citam que pode ocorrer interferência do estádio de desenvolvimento sobre a deposição de gotas de pulverização, uma vez que a germinação das plantas daninhas é irregular e distribuída no tempo, encontrando-se, em um mesmo dia, plantas em vários estádios e de vários tamanhos. Além de que, as pequenas dimensões das plantas daninhas e a posição destas no campo, estando sujeitas ou não à cobertura pela cultura, também podem intensificar a variabilidade dos depósitos unitários. Esses autores revelam ainda que ao realizar a padronização das plantas daninhas dentro do mesmo intervalo de massa seca ou de área foliar, nos diferentes posicionamentos a campo (linha e entrelinha), observam-se maiores depósitos nas plantas presentes na entrelinha em relação àquelas presentes na linha da cultura.

\section{CONCLUSÃO}

De forma geral, pode-se concluir que a quantidade e qualidade dos depósitos de calda de pulverização foram dependentes da espécie, do volume e da ponta utilizada, sendo que estudos com plantas em estádio distinto seriam importantes para um melhor entendimento do assunto.

\section{REFERÊNCIAS BIBLIOGRÁFICAS}

COSTA, E.A.D. Efeitos de surfactantes sobre a tensão superficial de soluções de rodeo. 1997. 73f. Dissertação (Mestrado em Agronomia/Proteção de Plantas)Faculdade de Ciências Agronômicas, Universidade Estadual Paulista, Botucatu, 1997. 
CUNHA, J.P.A.R. da; TEIXEIRA, M.M.; VIEIRA, R.F.; FENANDES, H.C. Deposição e deriva de calda fungicida aplicada em feijoeiro, em função de bico de pulverização e de volume de calda. Revista Brasileira de Engenharia Agrícola e Ambiental, Campina Grande, v.9, n.1, p.133-138, 2005.

FOLONI, L.L. Respostas da utilização de bicos de baixa vazão com glyphosate e sulfosate no controle de arroz vermelho, em aplicação de pré-plantio, em área de plantio direto de arroz (Oryza sativa L.). In: REUNIÃO DA CULTURA DO ARROZ IRRIGADO, 21., 1995, Porto Alegre. Anais... Porto Alegre, 1995. p.253.

HOLLOWAY, P.J. Surface factors affecting the wetting of leaves. Pesticide Science, v.1, n.1, p.56-63, 1970.

MARTINS, D. Deposição de calda de pulverização em cultivares de batata. 2004. 249f. Tese (Livre Docência em Agricultura/Produção Vegetal)-Faculdade de Ciências Agronômicas, Universidade Estadual Paulista, Botucatu, 2004.

NEGRISOLI, E.; TOFOLI, G.R.; VELINI, E.D.; MARTINS, D.; PALLADINI, L.A. Depósitos unitários de calda de pulverização com e sem surfactante em plantas de Salvinia molesta. Planta Daninha, Rio de Janeiro, v.20, p.51-56, 2002. Edição especial.
PALLADINI, L.A. Metodologia para avaliação da deposição em pulverizações. 2000. 111f. Tese (Doutorado em Agronomia/Proteção Vegetal)-Faculdade de Ciências Agronômicas, Universidade Estadual Paulista, Botucatu, 2000.

SOUZA, R.T.; VELINI, E.D.; PALLADINI, L.A. Aspectos metodológicos para análise de depósitos de pulverizações pela determinação dos depósitos pontuais. Planta Daninha, Rio de Janeiro, v.25, n.1, p.195-202, 2007.

TOMAZELA, M.S.; MARTINS, D.; MARCHI, S.R.; NEGRISOLI, E. Avaliação da deposição da calda de pulverização em função da densidade populacional de Brachiaria plantaginea, do volume e do ângulo de aplicação. Planta Daninha, Rio de Janeiro, v.24, p.183189, 2006.

VELINI, E.D. Estudos e desenvolvimento de métodos experimentais e amostrais adaptados à matologia. 1995. 250f. Tese (Doutorado em Agronomia/Produção Vegetal)-Faculdade de Ciências Agrárias e Veterinárias, Universidade Estadual Paulista, Jaboticabal, 1995. 